\title{
e-Phaïstos
}

e-Phaïstos

Revue d'histoire des techniques / Journal of the history

of technology

VII-2 | 2019

La sauvegarde du patrimoine scientifique

\section{Fenêtres historiques sur les rôles des espaces verts en plan de ville : Curitiba au Brésil, Monterrey au Mexique}

Historical Windows on the Roles of Green Areas in City Plan: Curitiba, Brazil and Monterrey, Mexico

Maclovia Corrêa-Da-Silva et Maria Eugenia González-Ávila

\section{OpenEdition}

Journals

Édition électronique

URL : http://journals.openedition.org/ephaistos/5465

DOI : 10.4000/ephaistos.5465

ISSN : 2552-0741

Éditeur

IHMC - Institut d'histoire moderne et contemporaine (UMR 8066)

Référence électronique

Maclovia Corrêa-Da-Silva et Maria Eugenia González-Ávila, « Fenêtres historiques sur les rôles des espaces verts en plan de ville : Curitiba au Brésil, Monterrey au Mexique », e-Phaïstos [En ligne], VII-2 I 2019, mis en ligne le 03 octobre 2019, consulté le 06 novembre 2019. URL : http:// journals.openedition.org/ephaistos/5465; DOI : 10.4000/ephaistos.5465

Ce document a été généré automatiquement le 6 novembre 2019.

Tous droits réservés 


\section{Fenêtres historiques sur les rôles des espaces verts en plan de ville : Curitiba au Brésil, Monterrey au Mexique}

Historical Windows on the Roles of Green Areas in City Plan: Curitiba, Brazil and Monterrey, Mexico

Maclovia Corrêa-Da-Silva et Maria Eugenia González-Ávila

1 Au cours des dernières décennies, les villes et l'environnement sont devenues une source de préoccupation en tant que milieu de vie urbain. Une recherche commandée par Munich, en Allemagne, faite par l'Economist Intelligence Unit, a proposé des indices de villes vertes pour l'Amérique Latine. Le critère de classification géographique par agglomération socioculturelle a inclus le Mexique et le Brésil en Amérique Centrale et du Sud. Avec une intention consciente, ce groupe de professionnels a montré les stratégies politiques et culturelles des villes qui pourraient apprendre les unes des autres $^{1}$ (voir figure 1 ). 
Figure 1 : Amérique du Nord (Mexique), Centrale et du Sud (Brésil).

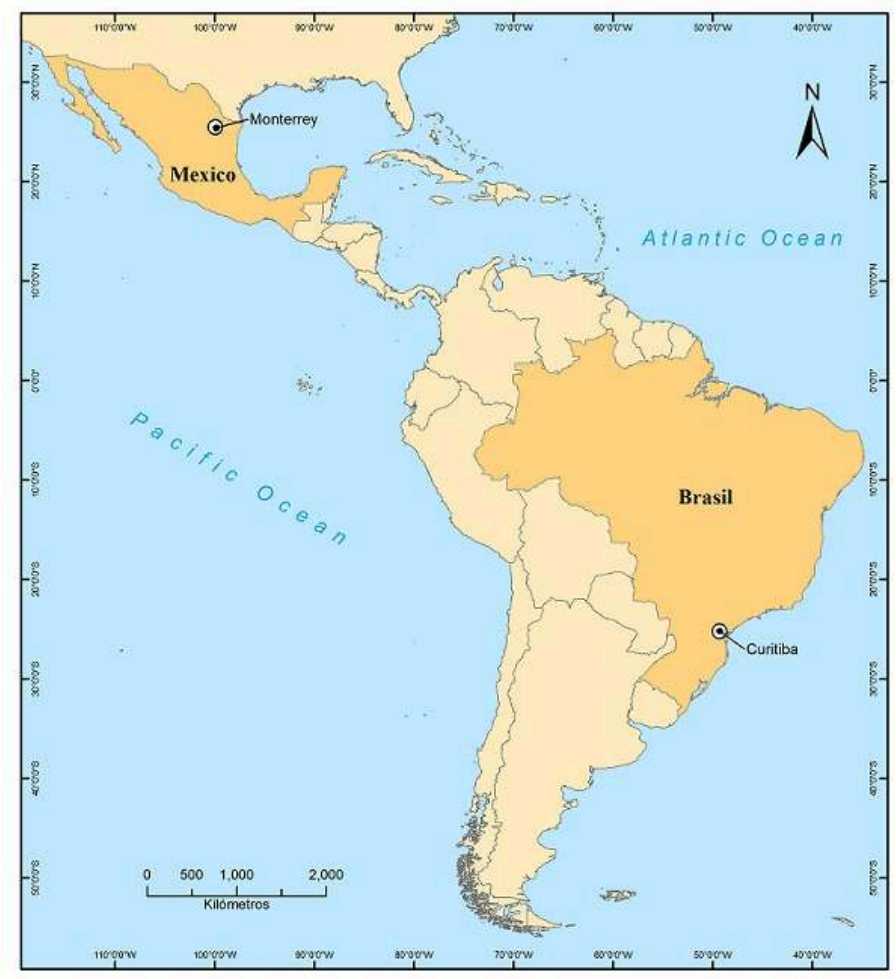

Crédit personnel des auteurs

Dans ce cadre, la ville de Curitiba se détache pour ses pratiques et ceci dans plusieurs catégories : énergie, transport, constructions, usage du sol, résidus, eau, qualité de l'air, assainissement, gouvernance environnementale. Par rapport à Monterrey et à d'autres villes, Curitiba a obtenu plus d'indicateurs supérieurs à la moyenne. Monterrey, par contre, partage ses indices verts avec la banlieue. Les auteurs, en tant qu'habitantes de ces villes, promeneuses au sein des espaces verts publics, ont posé la question suivante : qui sont les acteurs politiques responsables pour garantir leur intérêt, propreté et utilité ?

3 L'aménagement de territoires urbains au $\mathrm{XX}^{\mathrm{e}}$ siècle était sous-tendu par une forte relation avec le comportement naturel et social de l'être humain. Cette idée apparait clairement chez le théoricien de la cité-jardin, Ebenezer Howard, qui publiait en 1898 un projet de nouvelle société urbaine, sous le titre évocateur de Tomorrow ${ }^{2}$. La récupération des aspects culturels et sociaux de la campagne en ville était le but des concepteurs de ville.

\section{« Pourtant, l'incroyable popularisation du modèle urbanistique de cité-jardin a l'incontestable mérite d'obliger les habitants, les élus, les concepteurs à se préoccuper des relations de la ville avec la nature ${ }^{3}$ ».}

Renouveler la ville signifiait rationaliser son fonctionnement, prescrire des règles et un cadre pour sa croissance. Les professionnels européens et américains voyaient le plan comme un outil de travail pour comprendre et agir sur les territoires urbains. Le vecteur était social, réformateur, porteur de progrès pour l'humanité, en association avec la science et l'action politique et administrative : 


\begin{abstract}
«Accomplir cette mutation de la nature même de l'homme passe par une réorganisation à tous les niveaux, de la vie publique à la vie quotidienne ${ }^{4} »$.
\end{abstract}

$5 \quad \mathrm{Au} \mathrm{IV}$ Congrès International de l'architecture moderne, en 1933, à Moscou, Le Corbusier, un des principaux représentants du mouvement de l'architecture moderne, contribua à la rédaction du document connu en 1933 sous le nom de Charte d'Athènes. Cette charte introduisait le concept de ville fonctionnelle : habiter, travailler, circuler et recréer, d'une ville construite pour les citoyens, tout en sauvegardant le patrimoine historique. Les surfaces vertes devaient occuper une place définie soit dans les institutions, soit dans les habitations, pour séparer les bâtiments en hauteur ou pour la mise en valeur de la vie et du bien-être en ville.

6 Mais dans ces années 1930, la compréhension du plan d'urbanisme fut aussi influencée par la pensée organiciste qui provenait surtout des études écologiques et de la médecine. Le statut de ville organique renvoyait à un plan où la forme, la structure et le but étaient unis pour créer un environnement générateur de vie urbaine pour les citoyens et citoyennes. L'analogie naturaliste assimila la ville à un organisme physique vivant :

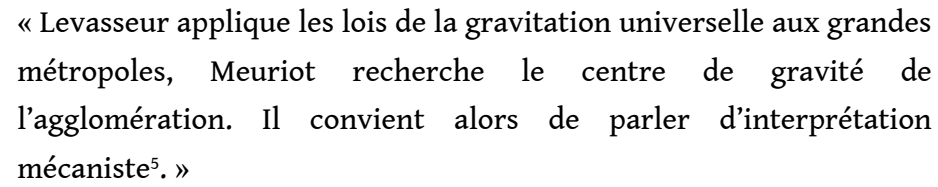

7 Opposé au fonctionnalisme de la ville radieuse de Le Corbusier, Gaston Bardet proposa un nouvel urbanisme avec les idées des quartiers-jardins : « Il doit être biologique ${ }^{6}$ ». La reconstruction des villes détruites par la guerre imposait des espaces verts, mais il manquait des parcs conçus comme une part de l'habitat.
« Les espaces verts des grands ensembles d'après-guerre n'ont guère tenu les promesses de la ville radieuse, mais, au-delà du discrédit qui les frappe aujourd'hui, l'idée qui les a inspirés mérite d'être comprise ${ }^{7} »$.

8 En Belgique, les plans d'aménagement apparurent à la fin de la Grande Guerre pour restructurer les communes dévastées. Mais l'aménagement des espaces au point de vue économique, social et esthétique, ne commença qu'en 1962. Les autorités cherchèrent par des plans de secteurs et zonage à conserver intactes les beautés naturelles et à protéger les sites du pays. Dans une zone de la ville de Charleroi, marquée par une forte présence des industries :

\footnotetext{
« L'objectif clairement affiché était de créer un lien entre Marchienne-au-Pont et le centre-ville en prévoyant, dans une vision à long terme, une conversion des zones les plus industrialisées en espace vert » (Araldo, 2010, p. 44).
}

Cela allait dans le sens d'une amélioration du cadre de vie et du renforcement de la centralité de la ville. En France, la loi d'Orientation foncière de 1967 orienta la croissance des nouveaux quartiers et la rénovation des espaces insalubres. Les schémas 
directeurs d'aménagement et d'urbanisme (SDAU), les plans l'occupation des sols (POS) français, en 2000, et la loi Solidarité et Renouvellement Urbain (SRU) ont été les outils pour envisager les défis de renouvellement urbain et de développement durable. La loi SRU fait le pont entre les politiques d'urbanisme, les politiques de mobilités et « la préservation de la qualité de l'air, de l'eau, du sol et sous-sol, des écosystèmes, des espaces verts, des milieux sites et paysages naturels ou urbains ${ }^{8}$ ».

Bernard Duraud observe néanmoins en 2012 que le renouvellement des conceptions de l'urbanisme ne s'est pas accompagné d'un développement social. Les inégalités demeurèrent, avec des classes populaires vivant aux alentours de la capitale alors que les fonctionnaires occupaient les espaces plus valorisées de la ville. Cela étant, un autre type d'aménagement n'assure pas l'absence des conflits et des inégalités. Avec l'accroissement du nombre d'habitants, les villes se modifient et se trouvent confrontées à la globalisation et à l'épuisement des ressources naturelles. Il y a actuellement une expansion urbaine très rapide, à l'échelle du monde, qui a pour conséquence un décentrement de l'urbain européen. Le Grand Paris est désormais la $25^{\mathrm{e}}$ ville du monde avec 12 millions d'habitants. Le décalage avec des villes de 35 millions d'habitants en Chine, des villes de 12 à 15 millions au Brésil, etc., est évident.

«Je ne fais pas l'éloge de la mégalopolisation. Je dis simplement que la question de l'urbain à l'échelle mondiale va extrêmement vite ${ }^{9}$.

Les grandes villes se sont transformées en environnements artificiels et dépendants de l'extérieur pour disposer de ce dont elles avaient besoin pour survivre. Les plus petites, malgré leur développement, ont conservé plus d'articulation avec la campagne. Étant donné l'importance des espaces publics verts dans les villes, il faut veiller à qu'ils ne disparaissent pas face à ces enjeux complexes.

«En général, il semble clair que si l'on ne satisfait pas les besoins fondamentaux d'êtres humains, le souci de l'environnement passe au second plan ${ }^{10} »$.

Globalement, l'écologie dans la ville a gagné en reconnaissance à la suite de la Conférence des Nations-unies sur l'environnement humain qui s'est tenue en 1972, à Stockholm, en Suède. Le Rapport Brundtland de 1987, intitulé « notre avenir à tous », et proposé par la Commission Mondiale sur l'Environnement et Développement de l'Organisation des Nations Unies a défini les conceptions du développement durable. Le développement durable, qui correspond à une prise de conscience à l'échelle planétaire, occupe une place prépondérante dans la volonté de sauver la planète, en évitant la destruction accélérée de la nature. En 1992, dans la ville de Rio de Janeiro, au Brésil, 178 chefs d'État ont signé une déclaration sur l'environnement, et, en parallèle, un plan d'action appelé agenda 21 , reposant sur 27 principes. Chaque pays, chaque ville, devait se mobiliser pour envisager des plans et des stratégies sous forme de programmes en faveur du développement durable.

\section{L'évolution des espaces publics : regard depuis les fenêtres}

« Tout d'abord, il y a les changements historiques très rapides qui interviennent sur le plan du monde urbain et de l'espace. Je vous rappelle que dans « espace », il y a le mot latin « spes » qui veut dire « 
espoir ». Dans ma manière d'organiser mon espace, qu'il soit privé ou public, c'est ma manière de regarder vers l'avenir ${ }^{11} »$.

Dans le plan de ville, la notion d'espace espace vert public, un espace non bâti, espace de recréation, de ventilation, d'aire libre, fut d'abord liée à la protection, l'hygiène et l'esthétique ${ }^{12}$. Mayté Banzo analyse le projet d'un parc linéaire aux portes de la ville de Lisbonne, idéalisé dans une dimension hygiéniste pour compenser le «tout urbain ». Mais l'excès de concentration de bâtiments transforma la dualité «urbain-rural en dualité urbain-structure verte $»^{13}$. L'auteure montre la variation synonymique de la terminologie dans les trois plans de ville pour la périphérie de Lisbonne. En 1964, le vert s'appelait «aires libres»; en 1992, "espaces libres critiques»; et, en 2002, " corridors vitaux ». On voit ainsi comment, dans les espaces urbains, ces espaces non bâtis accompagnent des discours esthétiques, protecteurs et environnementaux relatifs aux équilibres écologiques et humains.

Robert-Max Antoni montre dans le « séminaire Robert Auzelle » qu'il dirige, comment le thème des espaces publics s'est développé à partir d'un regard sur le "vide ». Il en conclut à la nécessité d'apprendre à regarder la ville. Le citoyen, un promeneur, s'approche du professionnel par le langage, et les deux proposent des solutions pour revitaliser les villes. Il a créé un vocabulaire de l'art urbain avec un chapitre sur la perception, et il détache deux termes fondamentaux pour aider les citoyens et les professionnels à regarder et à représenter la ville : «fenêtre urbaine (l'évolution dans les différentes époques sur le bâti, les voies et le tissu urbain) et séquence visuelle (une succession de vues associées à un plan en montrant comment l'une passe à l'autre) ». Les efforts des maires pour organiser les agglomérations ont porté d'abord sur les centres-villes et les quartiers limitrophes, qu'ils regardent depuis des fenêtres historiques renommées. Les représentations les plus fortes émanent des architectes, mais « il y a un point sur lequel tout le monde doit se retrouver. Il s'agit de l'espace libre, mais pas n'importe lequel : l'espace libre public ${ }^{14} »$.

15 L'espace public renvoie à l'espace politique (la mairie) et l'espace physique (les vides). L'un génère l'autre et le transforme en bien commun partagé entre les habitants. Le pouvoir local peut imaginer et mettre en œuvre des projets urbains pour transformer les zones inoccupées ou sous-utilisées des villes. Parfois, il est nécessaire de redynamiser un territoire pour améliorer le cadre de vie et atténuer la rareté foncière. La volonté politique de préserver des espaces naturels peut donc être mise en relation avec le maillage urbain serré.

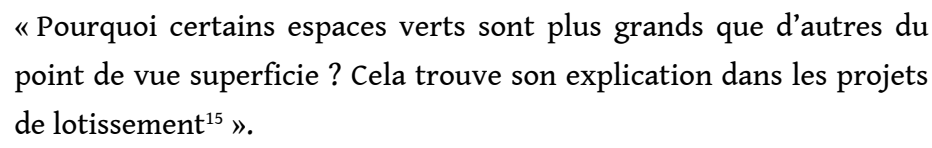

16 Marie-Clotilde Meillerand ${ }^{16}$, évoque, dans deux ouvrages, l'évolution de la notion d'espaces verts à Lyon, et sa liaison avec le zonage dès les premières tentatives d'aménagement du territoire. Elle ouvre la fenêtre des années 1940, où se développait la vie sociale organisée à partir d'un plan de ville. Le vert dans la ville était représenté sous la forme des jardins et parcs. Vers 1960, ces espaces naturels avaient la fonction d'offrir repos et liberté aux Citadins. La notion d'espace « vert » ou « libre » est encore étroitement lié à l'idée de " respiration ", en référence à la métaphore " organiciste " utilisée pour une des premières fois par Agache pour le plan de Rio, à la fin des années 
vingt. Cette notion est encore appliquée à la région lyonnaise au moment de son plan de 1946, avec la notion de " poumons verts » ${ }^{17}$.

17 Autre fenêtre : en Afrique, selon Tonde (1994), en plus des problèmes de la pollution, le phénomène de la désertification empire avec la dégradation de l'environnement. Pour lui, l'espace vert doit servir à réhabiliter et améliorer le cadre de vie des habitants. Il doit être accessible et offrir un cadre sain, des loisirs, ainsi que des activités culturelles, commerciales qui correspondent aux besoins des citoyens.

\section{S'équiper en espaces publics verts : les grandes villes brésiliennes}

Au Brésil, dans les années 1920, les autorités ont commencé à penser au développement industriel à partir de la capitale du pays, qui était alors Rio de Janeiro. La municipalité recruta l'architecte Donat-Alfred Agache pour élaborer le second plan de ville ${ }^{18}$, couvrant ses fonctions sociales, économiques et culturelles. La ville, comparée à un organisme parfait, ajoutait désormais à ses fonctions les mobilités, l'hygiène et l'aménagement.

19 Le document produit par Agache et son équipe n'a pas été discuté, diffusé ou même appliqué immédiatement et ceci pour des raisons politiques et financières ${ }^{19}$.

«Bien que peu de réalisations concrètes ont découlé de ce plan, son influence a été soutenue par les idées qu'il a permis de discuter (comme le zonage) et le premier grand code des travaux publié en 1937, qui continue à inspirer la législation actuelle en matière d'urbanisme $e^{20} »$.

Quelques idées ont été suivies au fur et à mesure que le champ de connaissances se solidifiait dans le pays. Les conceptions des années 1940, 1950 et 1960 montrent que le plan d'Agache a été repris par les maires suivants, qui ont investi dans des voies publiques, circulaires et radiales, tout en améliorant et prolongeant celles qui existaient.

\footnotetext{
"Malgré tout, beaucoup de ses propositions, bien que fragmentées, ont été prises en compte dans la nouvelle législation urbaine approuvée en 1937 (décret 6000 ) et dans des études ultérieures, par le Service Technique du Plan de Ville par José de Oliveira Reis. C'est le cas, par exemple, de l'urbanisation du Jardin d'Allah, clairement inspiré par les propositions d'Agache ${ }^{21}$ ”
}

Le projet urbain pour ériger la capitale du pays au centre de l'Amérique du Sud au cours des années 1950 a été conçu de manière à coïncider avec les espaces verts. L'architecte Lucio Costa a associé le fonctionnel et le moderne comme élément d'une nouvelle ville inscrite dans un lac artificiel. « De la croix est né l'oiseau de Brasília. La ville se construit autour de deux axes se croisant dont l'un est incurvé. Autour de ce squelette urbain cruciforme, c'est à Oscar Niemeyer que l'on doit le dessin des principaux édifices publics ${ }^{22}$ ». Les espaces libres, démocrates, amples et vides, avec de la pelouse et des plantes, devraient être contemplés et repris par la population. 
«La place centrale de la capitale devient partie d'un troisième et nouveau terre-plein, suivi d'un dernier où serait sauvegardée la végétation native du Cerrado ${ }^{23} »$. brésilien s'occupe du développement économique et des équilibres écologiques, en faisant appel aux dispositifs de planification et de gestion participative. Il n'a pas abandonné les outils de planification traditionnels, comme les codes, le zonage, les plans territoriaux locaux et les préceptes de l'urbanisme, mais pour ce qui concerne les politiques de l'environnement et le développement durable, il a investi dans des parcs naturels, réserves biologiques, stations écologiques et des opérations de reforestation ${ }^{24}$. et non bâtie dans les villes, assuraient deux choses : (a) une forme de développement, durable surtout, et (b) une valorisation forte pour l'immobilier. C'est un enjeu important pour l'avenir des villes de pouvoir disposer d'éléments qualitatifs qui préservent les caractéristiques paysagères et la convivialité dans des zones spécifiques. En respectant la régénération du milieu naturel et la protection de la vie, le plan de ville peut être le moyen d'y parvenir.

"Le concept de «villes vertes »- avec une capacité de récupération, d'autosuffisance et de durabilité sociale, économique et environnementale - est généralement associé à la planification urbaine dans les pays plus développés. Il indique une écoarchitecture de haute technologie, des routes bordées d'arbres pour les bicyclettes et le revenu et la bonne gouvernance pour tous les citoyens Cependant, il a une application spéciale et des dimensions sociales et économiques considérablement différentes dans les pays en développement à faible revenu. Là, les principes centraux des villes plus vertes peuvent guider le développement urbain qui garantit la sécurité alimentaire, le travail décent ${ }^{26}{ } \%$.

Autre point essentiel, la préservation des ressources en eau. Les diagnostics des sources indiquent qu'une intervention est nécessaire. Au Brésil, la Région Métropolitaine de São Paulo (RMSP) a souffert en 2012 d'un défi d'approvisionnement en eau pour 20 
millions d'habitants. Comprenant la ville de São Paulo et 39 autres municipalités, la ville et la banlieue occupent un territoire de 8000 kilomètres carrés qui concentre une forte croissance démographique et industrielle ${ }^{27}$. À ce problème s'ajoute celui de protéger les ressources hydriques, l'environnement, d'améliorer la qualité des logements. Les politiques urbaines accompagnent des phénomènes d'urbanisation qui menacent la végétation existante. L'Agence de planification Métropolitaine de l'État de São Paulo (EMPLASA), le Centre d'études de la métropole et d'autres entreprises et agences gouvernementales se livrent à un travail d'Hercule pour confronter les problèmes du développement technique et la dégradation de l'environnement qui s'est accélérée au cours des 50 dernières années. Selon la législation pertinente (article 8 , paragraphe $1^{\text {er }}$, de la Résolution CONAMA nº 369/2006).

«Il est considéré espace vert du domaine public, pour l'effet de cette Résolution, l'espace du domaine public qui joue le rôle écologique, paysager et récréatif, en favorisant l'amélioration de la qualité esthétique, fonctionnelle et environnementale de la ville dotée de végétation et d'espaces libre d'imperméabilisation. ${ }^{28}$ "

Karina Nascimento, qui a étudié 63 immeubles verts au nord du Brésil, dans la ville de Recife entre 1986 à 2007, constate une perte de 397214 mètres carrés de couverture végétale du fait des pressions immobilières ${ }^{29}$. Les problèmes principaux résident dans le choix des instruments fiscaux pour stimuler les propriétaires en vue de préserver les espaces verts. Située dans une région fragile, au bord de la mer, la ville de Recife souffre des conséquences des catastrophes naturelles et climatiques, lesquelles peuvent être atténuées par les espaces verts. Ce territoire de $218 \mathrm{~km}^{2}$ et 94 arrondissements possède d'autres immeubles dont les potentialités doivent déboucher sur la formation de couloirs écologiques, en partenariat avec la région métropolitaine et les autres villes qui font partie des Unités de Conservation de la Nature.

\section{Curitiba, Brésil, la fenêtre la plus verte}

Dans la ville de Curitiba, les immeubles qui conservent des aires vertes bénéficient de réductions d'impôts accordées par la mairie. En 1996, à travers la loi sur l'occupation des sols, les propriétés qui avaient préservé des espaces verts ont été intégrées dans les réserves particulières du patrimoine naturel de la Municipalité de Curitiba (RPPNM). Les propriétaires de ces espaces ont ouvert des fenêtres vertes, en modelant différentes ouvertures dans les quartiers. Aujourd'hui, ils peuvent proposer ces paysages à la communauté, garantissant ainsi une offre dans le domaine des activités d'écotourisme, d'éducation environnementale et de recherche scientifique.

Correspondant à une stratégie de préservation de portions de «binômes des forêts avec la forêt d'araucaria " en milieu urbain, la première RPPNM fait partie d'une organisation sociale destinée à assurer la qualité de vie dans un quartier de la zone ouest de la ville. Le commerce, les églises, les résidents et le club d'écologie ont fortement participé aux actions en vue de diminuer la contamination des deux rivières, augmenter les études de la flore et de la faune et préserver la biodiversité.

«Le RPPNM Cascatinha est né de l'effort des habitants du quartier de Santa Felicidade, dans la zone ouest de Curitiba. "Ici nous avons une caractéristique unique, d'union entre les canaux de communication 
et de représentation dans l'intérêt du voisinage", dit le propriétaire de la réserve, Eurico Borges dos Reis, qui y vit environ 30 ans ${ }^{30} »$. 1908359 en 2017. Le système de transports urbains a été aménagé à partir du plan de ville de 1965. Il soulève désormais des problèmes. Même si certaines lignes ne rivalisent pas directement avec les pistes de circulation automobile, les bus qui circulent dans des voies spécifiques ne rendent pas les services appropriés à la population et présentent un déficit de gestion.

\footnotetext{
«La décision de la ville de Curitiba de réajuster le taux de transport collectif avec un indice de plus du double de l'inflation devrait rendre encore plus difficile l'inversion de la tendance à la baisse du nombre total de passagers transportés par le système urbain. De 2011 à 2016, les dépenses totales de passagers sont passées de 246,8 millions par an à 211 millions, soit une réduction de $20 \%$. Et la projection est de nouveau en baisse jusqu'en $2017^{32}$ ».
}

Par contre, dans la ville, près de deux voitures par personne circulent. Les relations dynamiques entre les modes de transport, associées aux taxis, aux applications VTC, (Uber, LeCab, Chauffeur Privé33) qui roulent parallèlement, suscitent des déséquilibres. En effet, la diminution de la clientèle dans les transports publics est absorbée par les

e-Phaïstos, VII-2 | 2019 
usagers de voitures, qui encombrent les voies, provoquent des embouteillages et ralentissent le trafic ${ }^{34}$. Le niveau de pollution augmente. Pourtant, les bus qui circulent dans leurs propres voies (Bus Rapid Transit - BRT) peuvent transporter 270 personnes par voyage. Ils possèdent cinq portes et ont une longueur de 28 mètres. En 2017, la ville de Curitiba a reçu 25 véhicules neufs : «Le premier modèle, qui a commencé à circuler en 1991, a été développé précisément pour Curitiba ${ }^{35}$ ».

Deux pertes d'espace public ont marqué l'histoire de la circulation du BRT sur la ligne nord-sud. Le premier, dans les années 1990, a été caractérisé par la réduction de l'enceinte qui fermait l'accès du premier parc de Curitiba (fin XIX ${ }^{e}$ siècle, 1886), appelé «Passeio Público ». Pour élargir la rue Presidente Farias, les clôtures qui entouraient le parc furent retirées et le trottoir est devenu plus étroit. En outre, entre 2016 et 2017, un grand bâtiment construit en face du parc a provoqué un effet d'ombre pour les animaux, en hiver surtout.

Le deuxième a été occasionné par la circulation d'un grand bus autour d'une place. Le zonage et les règles municipales de construction ont engendré la perte d'une partie de la place du Japon (Curitiba) pour adapter le passage du bus BRT Ligeirão Nord-Sud. C'est un espace de loisirs avec de beaux arbres, très fréquenté par la population.

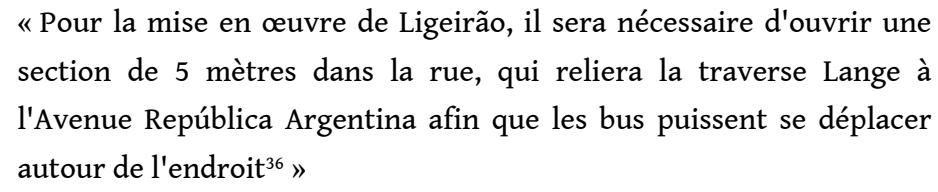

Les discours pour faire accepter ces réductions ont d'abord été économiques et technologiques : économie de 20 minutes sur le trajet de la ligne. Les 36000 usagers vont arriver plus tôt à destination. Il serait aussi possible d'atteindre l'équilibre des coûts d'exploitation du système de transport public. Les expressions économiques du développement, soit environnemental (la réduction de la pollution des gaz), soit social (confort, sécurité à l'embarquement et au débarquement, caméras de surveillance, des espaces pour les personnes handicapées), soit culturel (la vitesse réduite et programmée), soit intérêt politique ou d'autres, sont peu justifiées par la mairie relativement aux contraintes.
"Avec des slogans «S.O.S du Japon» et des ballons rouges dans les mains, les habitants de la place du Japon, Curitiba, se sont réunis dans une étreinte symbolique sur place ce dimanche matin. La démonstration vise à attirer l'attention sur les changements provoqués par la mise en œuvre de la nouvelle ligne Ligeirão sur le site. Selon les manifestants, le déménagement compliquera l'accès à la place et la rendra plus dangereuse, en particulier pour les personnes âgées et les enfants qui la fréquentent ${ }^{37}$ ».

Soares a constaté un fait semblable dans une ville du nord du Brésil, où la construction de quatre hauts bâtiments et d'autres plus petits, autour d'un espace vert, a séparé la végétation d'un parc des autres îlots verts qui existaient au sein de terrains bâtis. Plusieurs espaces où vivent des plantes et des animaux dans des zones protégées ont disparu ${ }^{38}$. 
«En 1977, la végétation du parc était déjà isolée des autres massifs verts trouvés au centre des quartiers, correspondant aux cours des lots à l'avant des routes. En 1998, il y avait quatre bâtiments dans la rue Nove de Janeiro et un dans l'Avenue Alcindo Cacela, qui a ombragé le Parc ZooBotanique ${ }^{39}$ ».

38 Le jardin Botanique de Curitiba a aussi eu sa végétation et des espaces pour piétons couverts par l'ombre des bâtiments. Les règles d'urbanisme dans les lotissements ont changé pour densifier certains espaces fonciers. Les permis de construire ont autorisé des constructions élevées. Le code de l'urbanisme a été modifié par l'autorité compétente, après l'accord de la majorité des habitants du quartier. Mais les votes n'ont pas été représentatifs de la majoritée ${ }^{40}$.

La place Miguel Couto, située dans le quartier nommé Batel, près du centre-ville, a aussi perdu ses caractéristiques originelles et une partie de sa superficie. Quand la famille du Baron du Serro Azul a donné ce terrain (fin du XIX ${ }^{\mathrm{e}}$ siècle), des plantes ont été cultivées et des liens affectifs se sont créés. Connue comme la «Pracinha do Batel» (Petite Place du Batel), de forme carrée et de petite taille (1 970 mètres carrés), elle a été coupée en deux portions par une voie. La mairie a justifié cette action par la nécessité d'améliorer la qualité du trafic.

Cet espace remarquable pour le citoyen abritait une foire gastronomique tous les samedis. Elle bénéficiait d'un environnement calme grâce à un faible trafic et la présence, depuis 1935, d'une cafétéria, d'un kiosque (Banca Batel), d'une station de taxis, ainsi que d'un traditionnel commerce de fleurs (Flora Pracinha do Batel). La mairie de Curitiba n'a pas souhaité préserver ce cadre malgré les arguments du mouvement d'amis du Quartier « Batel », coordonné par Rafael Xavier et d'autres citoyens.

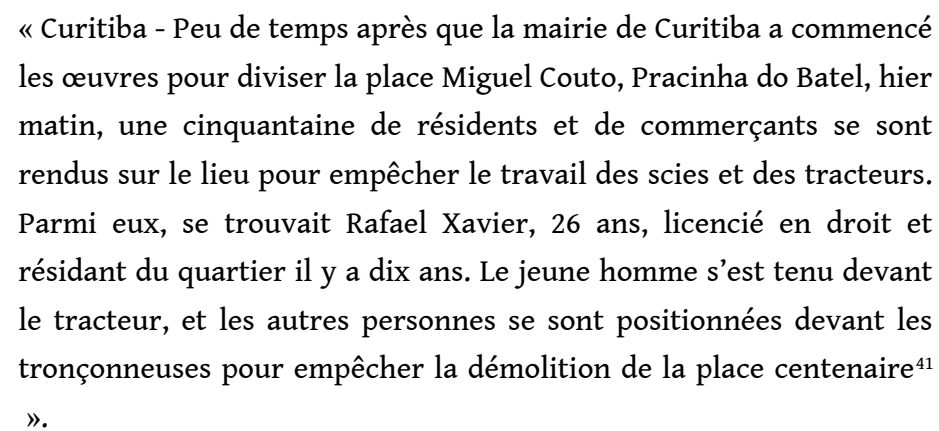

41 Quand cette manifestation pour la préservation du patrimoine a commencé, deux arbres étaient déjà coupés. La Justice (Bureau du procureur Municipal général) a empêché la poursuite des travaux pour analyser la légalité de ce dispositif de « revitalisation urbaine ». Le journal de la ville de Curitiba, Gazeta do Povo, s'est contenté de publier des informations sur le retard des travaux et le manque d'explications de la part des responsables. Ce même périodique évoquait aussi le fait que la Mairie avait gagné le droit de continuer les travaux et que la population gagnerait 294 mètres carrés comme extension de la place ${ }^{42}$. 
Figure 2 : Place du Japon et place Batel à Curitiba, Brésil.

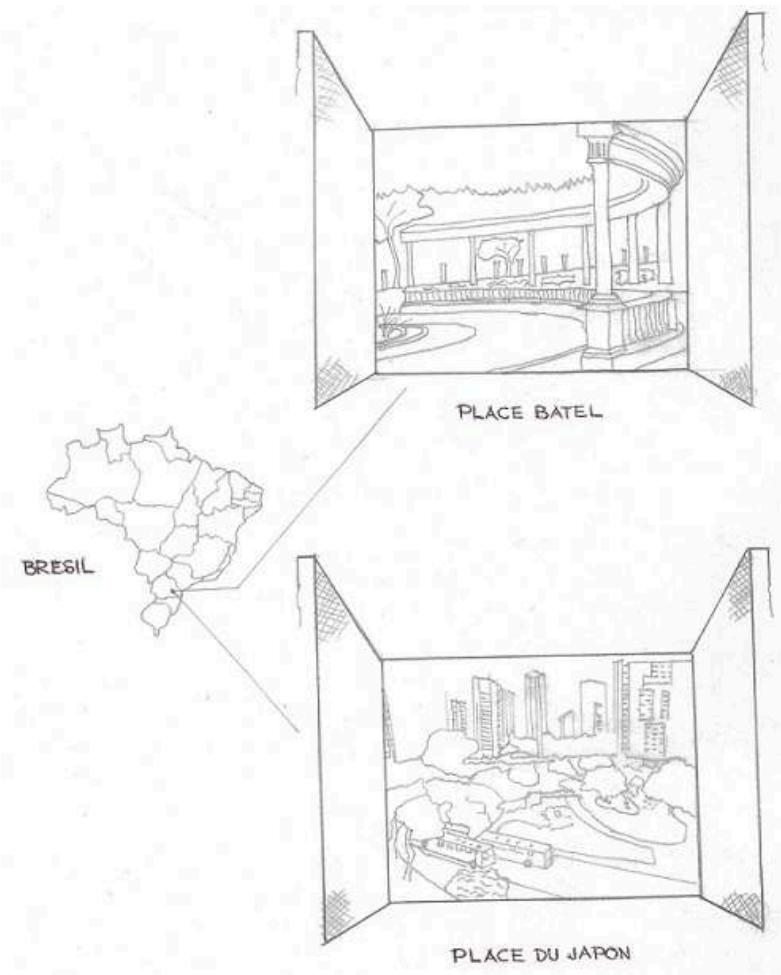

Crédit personnel des auteurs.

42 Le rôle des espaces verts comme endroits utilisés par les piétons ne fut pas pris en compte. Le percement du passage au milieu de la place Miguel Couto en faveur des voitures a été fermement justifié par les acteurs publics. La disparition de la verdure, la pollution de l'air, la détérioration de l'environnement et l'absence des principes de soutenabilité n'ont pas été prises en compte par les autorités locales. Cet espace vert était associé au commerce, à la qualité de vie et au loisir. Il faisait partie de la ville. Place du Japon, le patrimoine culturel et environnemental a été sacrifié et remplacé par une augmentation des émissions de gaz à effet de serre et par les dangers provoqués du fait de la circulation de bus de près de 30 mètres de longueur.

\section{La fenêtre historique de l'urbanisme au Mexique}

D'après C.J.J. Gutiérrez, en Amérique Latine, le processus d'urbanisation renvoie au courant développé en Europe au milieu du $\mathrm{XIX}^{\mathrm{e}}$ siècle et au début du $\mathrm{XX}^{\mathrm{e}}$ siècle. Figueroa fait référence à ces siècles comme une époque d'échange entre l'urbanisme français et l'urbanisme latino-américain ${ }^{43}$. Ces idées reprenaient surtout les travaux du Baron d'Haussmann à Paris, ville où dominait le développement de larges avenues ainsi que des espaces verts et ouverts ${ }^{44}$. Diane Davis ajoute que les modèles urbains développés dans les villes ont été appliqués de manière fragmentée et éparpillée dans le monde ${ }^{45}$.

Conformément aux études du professeur d'urbanisme Ricky Burdett, de la London School of Economics (LSE), dans son article «Inégalités et urbanisation »" , l'Amérique du Nord (73\%), l'Amérique du Sud (83\%) et l'Europe (82\%) sont les continents les plus urbanisés. 
Il pense que l'Afrique et l'Asie, en raison des indices de fécondité et des mouvements migratoires internes, vont accompagner ce processus au cours des prochaines décennies. Comme indiqué par L. Krishnamurthy et José Rente Nascimento ${ }^{47}$, le développement urbain dans les pays d'Amérique Latine et des Caraïbes, l'urbanisation en général, impliquent une plus grande consommation d'énergie et donc de ressources naturelles. Ces 20 dernières années, une série de changements ont affecté les statistiques de qualité de vie en ville, avec une plus grande vulnérabilité des populations qui vivent dans les zones rurales et urbaines, et la présence d'événements météorologiques extrêmes causés par le changement climatique mondial.

L'une des variables qui affectent le bien-être et la qualité de vie des populations est précisément celui des espaces verts qui font partie de l'aménagement urbain des villes et des métropoles du Mexique. Ricky Burdett dit que « dans de nombreuses parties du monde, l'urbanisation est devenue plus fragmentée dans l'espace et moins sensible à l'environnement, et elle accentue la fracture sociale ${ }^{48}$ ». Malheureusement, comme annoncé par L'Organisation de Coopération et de Développement Economiques, le Mexique vit un processus d'urbanisation rapide, contrairement à la plupart des pays qui composent l'OCDE ${ }^{49}$. En fait, en 2010, on estimait qu'environ $78 \%$ de la population vivaient dans des agglomérations urbaines ${ }^{50}$.

\section{Fenêtres historiques sur la planification et les espaces verts}

Bien que les idées d'urbanisme, au Mexique, se développent dès les premières décennies du siècle passé, il y a eu des interventions architecturales qui datent de l'époque coloniale, surtout à Mexico, la capitale du pays. Les dispositions de l'ordonnance de Felipe II, au XVI e siècle, réglaient la croissance de l'espace à partir d'une place carrée où étaient proposés des services pour répondre aux besoins vitaux. L'extension de la ville se faisait en ajoutant d'autres carrés à partir de cette place centrale. Cet aménagement a pris la forme d'un échiquier. La Plaza Mayor représentait l'espace géométrique de base, où une architecture civile unifiée et bâtie a été utilisée jusqu'au XVIII ${ }^{51}$.

Depuis la conquête espagnole du territoire mexicain au XVI ${ }^{\mathrm{e}}$ siècle, l'aménagement urbain ne s'est pas intéressé aux petites actions destinées à préserver les espaces verts. La dynamique écologique des zones lacustres, où la capitale était établie, n'a pas été comprise par les colonisateurs. La logique du pouvoir politique des conquérants reposait sur la déforestation des abords. Ce passage vers Mexico d'une zone lacustre à une logique d'élevage du bétail a conduit à un changement de la mécanique du bassin et de ses caractéristiques hydrologiques ${ }^{52}$. Plus tard, à la suite de l'indépendance vis-à-vis de l'Espagne en 1810, les terrains les moins dignes d'attention ont été transformés en espaces verts ${ }^{53}$.

Un autre type d'urbanisme, basé sur le néoclassicisme européen, a émergé au XIX ${ }^{e}$ siècle, au Mexique. L'influence française sur l'organisation territoriale de la capitale a introduit le plan d'urbanisme, développé par Ignacio de la Cantera. Celui-ci s'appuie sur les principes de symétrie, d'ordre, de régularité et de santé publique ${ }^{54}$. En 1910, la promenade de la capitale est inaugurée. Elle constitue une artère principale qui fête le centenaire de l'indépendance $\mathrm{du}$ Mexique. Sorte d'épine dorsale, cette voie commémore le centenaire de l'indépendance, mais aussi la fin du gouvernement de Porfírio Díaz (1876-1911) ${ }^{55}$. Ce président a lutté pendant plus de trente ans contre les 
invasions par d'autres nations et pour la liberté du pays. Il a soutenu l'Église catholique, l'université, la banque nationale, les exportations des matières premières et l'École militaire navale. La Révolution Mexicaine de 1911 a mis fin au pouvoir dictatorial de Diaz.

Les idées de l'urbanisme français étaient approuvées par Diaz. Elles correspondaient au goût des architectes mexicains et à celui de l'ingénieur José Antonio Cuevas, qui remarquait la prolifération des bâtiments et des puits artésiens ; d'Adolfo Zamora, qui défendait la maison multifamiliale au lieu de l'unifamiliale; de l'ingénieur Miguel Ángel de Quevedo, qui suggérait des boulevards, des parcs et des jardins pour les villes. Quant à Luis Sánchez Ponton, il se montrait partisan des idées interdisciplinaires en faveur de l'urbanisme et des plans de villes afin de disposer d'outils multiples pour promouvoir des conditions de vie plus humaines ${ }^{56}$.

Carlos Contreras, grâce à sa formation reçue aux États-Unis, a introduit des concepts fonctionnels d'hygiène et d'ordre pour aménager le territoire ${ }^{57}$. L'architecte Contreras affirmait que « doter la ville d'espaces verts était essentiel et faisait partie de son projet de régulation de la croissance et de sa vision de la planification de l'expansion urbaine à venir ${ }^{58}$ ». Quand, vers 1920 , Contreras a commencé à travailler à Mexico, le District fédéral (DF), il y avait un département des travaux publics qui approuvait la construction de bâtiments sans aucun plan de ville. L'architecte a posé les bases de l'urbanisme, en établissant des théories, une méthodologie, accompagnées de lois et de règlements.

51 Dans les plans proposés par Contreras pour le District Fédéral furent, au cours des années, adoptés: les circuits intérieurs et extérieurs, les grandes avenues qui le traversaient (par exemple, les routes), la rue principale, qui serait l'avenue San Juan de Letrán (de Tlalnepantla au début de l'autoroute à Cuernavaca) qui a fini par être Insurgentes, le métro, avec, en 1949, les premières lignes, et l'urbanisation d'El Pedregal, l'œuvre de l'architecte Luis Barragán, dont il a dessiné le fractionnement sur des photographies aériennes ${ }^{59}$.

52 Mais l'industrialisation nationale a eu un impact négatif sur la conception du plan et la soutenabilité de l'urbanisation urbaine. Face à la croissance démographique accélérée, favorable à l'adoption d'un modèle expansionniste de croissance urbaine, les dirigeants ont été confrontés à des problèmes de gouvernance et d'administration ${ }^{60}$. Alfonsin et Fernandes expliquent que, dans les villes mexicaines des années 1970, le nombre moyen de normes pour créer des lotissements et constructions était de 30. Mais ces normes ne s'intégraient pas obligatoirement dans un plan d'aménagement du territoire.

En 1976, la question de l'urbanisme a été abordée par la Loi générale des établissements humains: "Ley General de Asentamientos Humanos" - LGAH ${ }^{61}$, qui a encouragé l'élaboration d'autres lois, de plans de ville, et des règlements d'ordonnance territoriale pour plusieurs villes mexicaines et institutions. Deux ans après, en 1978, une autre loi a donné davantage de libertés aux autorités gouvernementales en vue d'aménager les territoires urbains ${ }^{62}$.

54 Plus tard, au milieu des années 1980, le Système national de planification démocratique pilote les questions de développement économique et urbain sur la base des mesures déjà prises. Il poursuit cette mission jusque dans les années 1990. Les investissements dans le cadre du programme "Villes moyennes " se sont bornés à une meilleure maitrise de la croissance urbaine, sans vision globale et sans réflexion conceptuelle. Selon Gutiérrez, ce cadre entérine le maintien de cette position pendant 30 ans, sans 
disposer des moyens de satisfaire les besoins sociaux et environnementaux d'un grand nombre de citoyens ${ }^{63}$. possédait 528168 hectares d'aire verte, en 2004, contre 1839,75 hectares couverts par le vert en 2013. Selon ces auteurs, qui ont réalisé une interprétation visuelle des images satellites, la couverture végétale de la ville n'est pas obligatoirement accessible à la population, alors qu'un espace vert offre toujours des bénéfices aux citoyens. Il favorise la biodiversité, le contrôle des inondations et l'intégration sociale. Par conséquent, il y a, dans la ville, $6,2 \mathrm{~m}^{2}$ d'espaces verts par habitant. Ce résultat ne peut être interprété comme supérieur aux 4,8 $\mathrm{m}^{2}$ rapportés par Roccatti (2004) car il faut tenir compte du fait que la plupart des espaces verts se concentrent dans le sud et l'ouest de la ville, ce qui les rend pratiquement inaccessibles aux habitants du centre et du nord ${ }^{67}$.

Pérez, Cuéllar et Treviño ont aussi remarqué le manque de plans et de politiques urbaines pour créer d'autres espaces verts, tout au long du XXe siècle. Pour les maires des villes, les mesures prioritaires résidaient dans la conservation et préservation de la qualité de ces espaces et le contrôle de l'extension du territoire par rapport à la croissance de la ville. Les auteurs établissent le lien entre la croissance de la population, la diminution du paysage vert ainsi que le manque d'articulations entre les plans de ville et les espaces verts. Les villes qui ne recherchent pas d'équilibre entre les espaces bâtis et les aires libres sont désordonnées. Cette réalité prit tellement d'envergure qu'il est presque impossible de modifier la situation actuelle dans certains espaces urbains : 
« sa configuration initiale et l'absence d'un plan de développement initial rendent pratiquement impossible la création de nouveaux espaces verts ${ }^{68} »$.

Sánchez Ruiz signale qu'avec la formation de l'Association Nationale pour la planification de la République Mexicaine, en 1926 et des lois qui se sont ajoutées aux actions sur le territoire urbain, certaines villes ont bénéficié d'un élan. Monterrey, par exemple, a créé la loi de planification et des règlements en 1927. Elle a conduit à des interventions municipales en faveur de l'embellissement du centre-ville ${ }^{69}$. L'architecte Carlos Contreras a travaillé à partir de supports qui prévoyaient le développement ordonné selon la topographie, le climat et les fonctions urbaines, sociales comme économiques.

61 Pour réaliser l'un des objectifs de la planification, une certaine forme de contrôle fut jugée nécessaire sur la propriété publique et privée. Jusqu'à ce moment, il n'y avait qu'une première loi pour Monterrey, préparée par Carlos Contreras, quand Aarón Sáenz était gouverneur de Nuevo León ${ }^{70}$. Les lois sont des outils fondamentaux pour mettre à exécution les plans de travail d'urbanisme. Ce sont des éléments importants de la pensée pragmatique des villes industrielles.

Les usines se sont installées au bord des rivières, l'eau étant un intrant important pour la production, affirme Perez Daniel :

« Les rivières servaient aussi de décharges; de nombreuses rivières ont disparu en raison de l'activité industrielle, comme la rivière Santa Catarina à Monterrey ${ }^{71} »$.

Vers 1933, les activités de cette grande fonderie (Fundidora), au bord de la rivière Santa Catarina associées à une grande usine de bière (Cevecería Cuauhtémoc), s'est traduit par l'expansion des maisons ouvrières. Les quartiers les plus importants se situaient près des industries. Ces espaces d'habitats se mélangeaient aux routes, aux trains et aux vides immobiliers. Les ouvriers vivaient aux limites de la ville, au nord et au sud, ou au centre-ville. Les espaces occupés par les riches se sont peu à peu détériorés. Aujourd'hui, la rivière Santa Catarina souffre des déchets et de la déforestation sur son bassin versant ${ }^{72}$.

Perez Daniel, qui décortique les discours sur la modernité, remarque que la sphère publique a été responsable de la construction des espaces urbains aménagés, mais que la modernisation s'est maintenue en dépit des pratiques politiques. En 1861, le gouvernement avait créé l'Alameda Escobedo, avec une végétation exubérante, un espace pour marcher, se promener et rencontrer des gens. Casillas Zapata, Elizondo et Moreno $^{73}$ remarquent que ces espaces ont représenté les premiers terrains ouverts d'interaction sociale. Cependant, en 1886, la moitié de l'espace était occupé par un pénitencier et les autres partis du terrain avaient été vendus. Les mêmes auteurs observent qu'en plus de 50 ans, plusieurs places se trouvèrent réduites en superficie, du fait de la construction de bâtiments publics (voir tableau 1).

Tableau 1 : Ville de Monterrey.Perte des espaces ouverts et verts (1886-1950)

\begin{tabular}{|l|l|l|}
\hline $\begin{array}{l}\text { Intervalle } \\
\text { temporel }\end{array}$ & $\begin{array}{l}\text { Espaces publics de Monterrey } \\
\text { modifiés }\end{array}$ & Les réalisations spatiales par les maires \\
\hline
\end{tabular}




\begin{tabular}{|l|l|l|}
\hline 1886-1909 & $\begin{array}{l}\text { Alameda Mariano Escobedo. Plaza } \\
\text { de Colón, Place Zuazua } \\
\text { Place 5 Mai et Place Juárez }\end{array}$ & $\begin{array}{l}\text { Pénitencier ; agence de voitures, marché Colón, } \\
\text { marché Juárez, Palais du Gouvernement et } \\
\text { École Primaire }\end{array}$ \\
\hline $1928-1950$ & $\begin{array}{l}\text { Place de la République; Place San } \\
\text { Jacinto ; Place la Purísima et Place } \\
\text { Bolívar }\end{array}$ & $\begin{array}{l}\text { Modifications et ampliation des espaces } \\
\text { routiers, École Primaire }\end{array}$ \\
\hline
\end{tabular}

Source : Casillas Zapata et al., 2018, p.14-15. présent protégés comme leurs prédécesseurs, mais le 21 Décembre 2011 a été publié dans le Journal officiel le décret d'extension du parc urbain La Pastora qui va augmenter de 13,65 hectares situés principalement dans les rives de la rivière La Silla, la seule rivière vivante dans la région métropolitaine. [...] il a accordé une concession à FEMSACoca-Cola pour occuper une superficie de 26 hectares de cette zone pendant 60 ans pour bâtir le stade de football du Club Los Rayados, une concession impossible d'être contestée par le mouvement écologiste [...], laquelle a causé des difficultés aux cours d'eau de la rivière La Silla et d'agrandir le déficit en espaces verts ${ }^{75}$. Coca Cola Femsa est aussi présent dans les espaces du Parc Fundidora. Fermée en 1986, l'industrie du fer et d'acier a connu une série de plans directeurs pour créer un parc écologique inauguré en 2001. Le Parc, selon la presse locale, a perdu ses caractéristiques d'espace public dans la mesure où il offre des amusements payants, accessibles à ceux qui ont un pouvoir d'achat élevé ${ }^{76}$.

En 2015, avec plus de 1110000 habitants et même 4,5 millions pour la grande Monterrey (Région Métropolitaine de Monterrey), la ville abrite, à la place de la fonderie, une superficie de 144 hectares, dont 80 hectares sont des espaces verts, avec 14418 arbres, 23 sources d'eau, deux lacs et quatre terrains de stationnement payant. Des attractions payantes ont fait leur apparition avec le carrousel, la roue de la fortune, les bateaux du lac, les vélos, la Cineteca, les musées, et le patinage sur la glace. À l'intérieur du parc, le consommateur paye des frais supplémentaires. Divertissements culturel, sportif, éducatif, écologique et commercial sont les fonctions du parc, et selon

e-Phaïstos, VII-2 | 2019 
les parlementaires de l'État du Nuevo León, « le parc Fundidora en tant que poumon de notre zone métropolitaine » représente un espace vert majeur ${ }^{77}$.

Figure 3 : Parc Fundidora (FEMSA-Coca-Cola) et Parc La Pastora qui abrite le Stade de Football à Monterrey, Mexique.

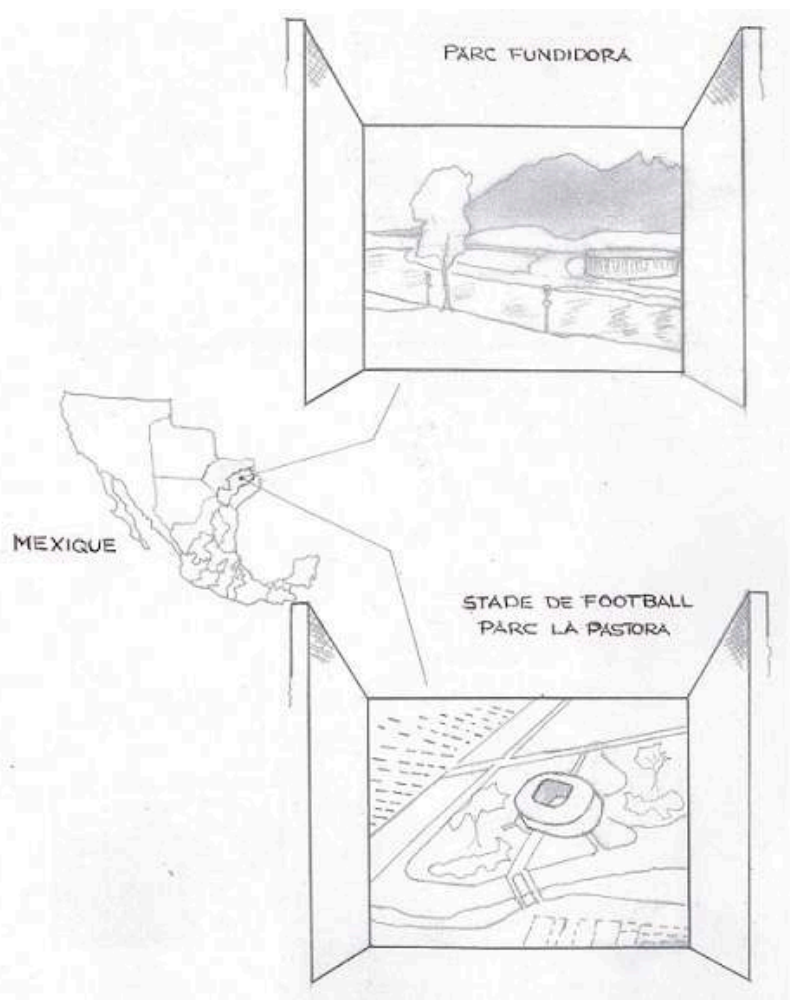

Crédit personnel des auteurs.

\section{Fermer les fenêtres...}

Les politiques urbaines et l'urbanisme $\mathrm{du} \mathrm{XX}^{\mathrm{e}}$ siècle sont fortement liés à l'aménagement du territoire des villes par des plans de ville. L'idée de plans organiques, dans la perspective de l'organisation rationnelle des organes physiques et de la structure de fonctionnement du corps humain, a prévalu sur toutes les autres. Les volets environnementaux : l'air, l'eau et le sol, à partir des regards hygiénistes, sont perceptibles dans la forme des places, parcs et jardins publics, qui se sont peu à peu transformés en espaces marqués par de forts enjeux politiques. Le désintérêt environnemental transparaît à travers les conflits et alliances autour des projets de régénération urbains concentrés dans les mains des architectes et ingénieurs, surtout au niveau des centres-villes.

Nous sommes d'accord avec Charles Wright Mills (1916-1962), sociologue américain, quand il mentionne que le rationalisme n'assure plus la liberté ni la démocratie. La partie centrale de cette idée réside dans le manque de responsabilité des intellectuels envers les masses populaires, et le contrôle social des oligarques. D'autres auteurs nous ont aidé à établir des relations entre les concepts et les discours sur les espaces verts entre deux villes, confrontées à la fois à des industries et à des réserves biologiques en milieu urbain. 
70 l'érosion progressive des valeurs de la démocratie, de la culture et du pacte social. Les rapports avec la réalité étaient éloignés de l'interprétation historico-politique la plus répandue dans la société. Il n'y avait pas d'efforts pour réfuter cette pensée dominante progressiste.

71 Les espaces verts disponibles ont désormais acquis différentes fonctions, comme apporter un équilibre, la biodiversité. Mais quel imaginaire garantissent les discours sur la durabilité et le patrimoine naturel, un imaginaire maitrisé par la nature ou l'écologie, ou un imaginaire maîtrisé par l'être humain? Nous, comme habitants et promeneurs au sein de ces villes, nous pouvons déplacer les limites de ces fenêtres historiques avec nos droits de citoyen et constater les nuances des orientations politiques et des arrangements institutionnels pour intervenir sur des projets urbains.

Travailler sur les représentations de la nature dans les villes, au profit de l'extension urbaine, implique d'éliminer les fragmentations entre les diverses conceptions du territoire et les usages. De quelle manière les législateurs pourront-ils répondre aux interrogations collectives si les appareils administratifs centralisent le pouvoir? Il faut que la population accède aux postes de représentation dans les quartiers pour éviter les actions qui, progressivement, se feraient au profit exclusif de certains groupes. Faire mordre la poussière aux plans de ville, c'est comme fermer les yeux sur les techniques d'orientation et de transformation des espaces verts ${ }^{78}$.

PINTO Monica, Ambiente Brasil (2007). Exclusivo : Curitiba tem a primeira Reserva Particular do Patrimônio Natural Municipal-RPPNM do país, 2007, http://noticias.ambientebrasil.com.br/ exclusivas/2007/11/20/34785-exclusivo-curitiba-tem-a-primeira-reserva-particular-dopatrimonio-natural-municipal-rppnm-do-pais.html.

\section{BIBLIOGRAPHIE}

ANTONI Robert Max (dir.). De l'espace public. Séminaire Robert Auzelle. Art Public. Ministère du Logement et de l'habitat durable, 2015, https://www.arturbain.fr/arturbain/dossiers/documents/ G_2016_de_1_espace_public.pd.

ARALDO Filippi. Les politiques de densification urbaine, utopie ou réalité ? Master en Sciences et Gestion de l'Environnement, Université Libre de Bruxelles, 2010.

AURICOSTE Isabelle. « Urbanisme moderne et symbolique du gazon. » Communications, $\mathrm{n}^{\circ} 74,2003$, p.19-32.

AZUELA Antonio. "Sobre las nuevas condiciones en el acceso al suelo y en la regulación urbana en México », dans FERNANDES Edésio, ALFONSIN Betânia (dir.). Direito urbanístico. Estudos brasileiros e internacionais, Belo Horizonte Del Rey, 2006, p.111-138.

BANZO Mayté, «Structure verte et mise en cohérence de la périphérie métropolitaine de Lisbonne ", Revue géographique des Pyrénées et du Sud-Ouest, 2007, p.109-124.

e-Phaïstos, VII-2 | 2019 
BAZANI Adamo. Volvo confirma a entrega de 25 novos biarticulados para Curitiba em março, Diario do Transporte, 2017, https://diariodotransporte.com.br/2017/12/27/volvo-confirma-a-entregade-25-novos-biarticulados-para-curitiba-em-marco/.

BERDOULAY Vincent, « Modernismo e espaço público: o Plano Agache do Rio de Janeiro », Revista Território, Ano VII, n¹1, 12 et 13, 2003, p.123-132.

BURDETT Ricky, Inégalités et urbanisation, 2016, www.oecd.org/fr/regional/inegalites-eturbanisation.htm.

CASILLAS ZAPATA, Amanda Melissa, ELIZONDO María Teresa Ledesma, MORENO Carlos Estuardo Aparicio, «Conformación de áreas verdes y espacios abiertos en la transformación urbana de Monterrey del siglo XVII a inicios del siglo XXI », Letras Históricas, 2018, www.letrashistoricas.cucsh.udg.mx/index.php/LH/article/download/6728/6305, p.1-32.

CONGRESO DEL ESTADO DE NUEVO LEÓN, Punto de acuerdo para solicitar comparecencia de director del parque fundidora, 2016, www.hcnl.gob.mx/glpvem/2016/10/punto-de-acuerdo-para-solicitarcomparecencia-de-director-del-parque-fundidora.php.

DAVIS Diane, El Leviatán urbano. La ciudad de México en el siglo XX, Mexico, Distrito Federal, Fondo de Cultura Económica,1999.

DOURADO Anisio Brasileiro de Freitas, « Développement urbain et transports collectifs au Brésil. Les enseignements de l'expérience de Recife et de Curitiba », Sociologie, 1991, https:// pastel.archives-ouvertes.fr/file/index/docid/529422/filename/ 1991TH_BRASILEIRODEFREITASDOURADO_A_NS15716.pdf

DUBARLE Patrick, PELLEGRIN Jean-Pierre, Politiques et pratiques d'aménagement du territoire au Brésil, Paris, Datar, 2009-2010, https://www.aeidl.eu/images/stories/pdf/datarbresil.pdf

DUBOST Françoise, LIZET Bernadette, « La nature dans la cité », Communications, $n^{\circ} 74,2003, p$. 5-18.

DURAUD Bernard, « Et soudain Brasilia bouleversa l'urbanisme », L'Humanité.fr, 2012, www.humanite.fr/culture/et-soudain-brasilia-bouleversa-l-urbanisme-510484.

EL HORIZONTE, Acaparan privados áreas verdes de Parque Fundidora. Los directivos del parque estatal elevaron la frecuencia de eventos privados en la zona de las S's, que es su principal área verde, 2016, http://www.elhorizonte.mx/local/acaparan-privados-areas-verdes-de-parque-fundidora/ 1668391.

EL HORIZONTE, Parque Fundidora hace elitista diversión familiar. Con precios elevados, proveedoras de entretenimiento hacen su agosto a costa de la economía familiar, 2014, http://www.elhorizonte.mx/ local/parque-fundidora-hace-elitista-diversion-familiar/838887.

ESCUDERO Alejandrina, « Carlos Contreras : primer urbanista del siglo XX en México » Revista Digital, 2007, http://discursovisual.net/dvweb08/diversa/divsemale.htm.

ESCUDERO Alejandrina, SÁNCHEZ RUIZ, Gerardo G GARZA, Carlos Ríos, « Estudios Introductorios », Revista Planificación (1927-1936), Universidad Nacional Autónoma de México, 2018, http://fa.unam.mx/editorial/wordpress/wp-content/Files/raices/RD07/contenidos/ ESTUDIO_INTRODUCTORIO.pdf.

ESPARZA HERNÁNDEZ, GERARDO Luis, Estudio del manejo y conservación del parque nacional Cumbres de Monterrey (PNCM), según decreto del 2000 bajo un enfoque de desarrollo sustentable basado en la participación ciudadana, Universidad Autónoma de Nuevo León, Instituto de Investigaciones Sociales, 2014, http://eprints.uanl.mx/4057/; http://cdigital.dgb.uanl.mx/te/ 1080253674.PDF. 
FARIA Renata Pereira, «Plan Agache de Rio de Janeiro, capital federal de Brasil : urbanismo de excelencia en los años 20 ", Urbano, vol.9, n¹3, p.78-81, 2006, http://docplayer.es/68076267Urbano-universidad-del-biobio-issn-version-impresa-chile.html.

FIGUEROA Jonás, La arquitectura y el urbanismo en la América Latina del siglo XX, Valencia, En Puncel Alfonso (Ed.), Universitat de Valencia, 1994.

MORADORES IMPEDEM OBRAS NA PRAÇA DO BATEL, Folha de Londrina, 2007, https:// www.folhadelondrina.com.br/cidades/moradores-impedem-obras-na-praca-dobatel-605274.html.

GUTIÉRREZ C.J.J, « Planeación urbana en México : un análisis crítico sobre su proceso de evolución urbano », Universidad del Bío Bío, Vol.12, n¹9, 2009, p.52-63.

ÍNDICE DE CIDADES VERDES DA AMÉRICA LATINA, Avaliando o desempenho ambiental das principais cidades da América Latina. Projeto de pesquisa realizado pela Economist Intelligence Unit, patrocinado pela Siemens, 2010, https://www.siemens.com/entry/cc/features/ greencityindex_international/br/pt/pdf/report_latam_pt_new.pdf.

INSTITUTO NACIONAL DE ESTADÍSTICA Y GEOGRAFÍA, Principales resultados del Censo de Población y Vivienda 2010, http://internet.contenidos.inegi.org.mx/contenidos/Productos/ prod_serv/contenidos/espanol/bvinegi/productos/censos/poblacion/2010/princi_result/ cpv2010_principales_resultadosI.pdf.

HASSLER Márcio Luís, A natureza na cidade: Uma abordagem a partir da percepção da população acerca do Jardim Botânico de Curitiba-PR, Mestrado em Geografia., Universidade Federal do Paraná, 2006, https://acervodigital.ufpr.br/bitstream/handle/1884/3934/marcio\%20hassler.pdf.

HORTICULTURA URBANA Y PERIURBANA, ORGANIZACIÓN DE LAS NACIONES UNIDAS PARA LA ALIMENTACIÓN Y LA AGRICULTURA. CIUDADES MÁS VERDES, ¿Ciudades de sufrimiento o de oportunidades?, 2018, http://www.fao.org/ag/agp/greenercities/es/hup/index.html.

KRISHNAMURTHY L., NASCIMENTO José Rente (eds.), Áreas Verdes Urbanas en Latinoamérica y el Caribe, 1998, www.rivasdaniel.com/Pdfs/Areas_verdes_LatAmerica.pdf.

LXXIV H. Congreso del Estado de Nuevo León. (2016). Comparece director de Parque Fundidora,http:// www.hcnl.gob.mx/sala_de_prensa/2016/11/comparece_director_de_parque_fundidora.php

LUCIO COSTA, Le véritable père de Brasilia, Lumière de la ville, https://lumieresdelaville.net/luciocosta/, 2016.

MARCHIORI Raphael, BORDIN Laura Beal, « Em 5 anos, Curitiba perdeu 20\% dos passageiros de ônibus; tendência é piorar ", Gazeta do Povo, 2017, https://www.gazetadopovo.com.br/vida-ecidadania/em-5-anos-curitiba-perdeu-20-dos-passageiros-de-onibus-tendencia-epiorar-7qyt68u2nls4uxsmvgomombaf.

MCMICHAEL C, « The Urban Development of Mexico City,1850,1930 », Planning Latin America's Cities, 1850-1950, 2002.

MEILLERAND Marie-Clotilde, Les espaces verts dans les plans d'urbanisme de l'agglomération lyonnaise : des espaces verts à la ceinture verte, Lyon, ENS-LSH, Séminaire GRAIQUE, 4 mars 2008, http://pradis.ens-lsh.fr/.

MONCEF Btissam, CARBONE Valentine, SOULEROT Marion, Le management durable au cour des organisations, Paris, Lavoisier, 2011. 
MONGIN Olivier, Conférence sur l'élaboration du plan local d'urbanisme. Intégral du discours de M. Mongin, tenu le 6 octobre 2010 à l'Hôtel de Ville de Neuilly-sur-Seine, https:// www.neuillysurseine.fr/files/neuilly/mairie/plu/discours-olivier-mongin.pdf.

MONTIGNY Gilles, De la ville à l'urbanisation, Paris, Éditions L'Harmattan, 1992.

MORADORES ABRAÇAM, « A praça do Japao em protesto contra novoligeirao », Gazeta do Povo, 2018. Disponible à l'adresse : https://www.gazetadopovo.com.br/curitiba/moradores-abracam-apraca-do-japao-em-protesto-contra-novo-ligeirao-evau9hiz6frkioel9xpl663yh.

NASCIMENTO Karina Barros Freitas, Áreas verdes e clima urbano: a função socioambiental dos imóveis de proteção de áreas verdes na cidade - Recife/PE, Brasil. Dissertação (Mestrado), Programa de PósGraduação em Desenvolvimento Urbano, Universidade Federal de Pernambuco, Recife, 2011, https://repositorio.ufpe.br/handle/123456789/3027.

OCDE, Estudios de Políticas Urbanas de la OCDE : México Transformando la Política Urbana y el Financiamiento de la Vivienda, 2015, https://www.oecd.org/gov/sintesis-del-estudio-mexico.pdf. PAQUOT Thierry, « Ville et nature, un rendez-vous manqué ?», Diogène, n²07, 2004, p.83-94.

PEREZ DANIEL Gustavo Héron, Estudios de Historia Moderna y Contemporánea de México, $\mathrm{n}^{\circ} 42$, juliodiciembre 2011, p.75-108.

PÉREZ Javier Jiménez, CUÉLLAR Geraldo, TREVIÑo Eduardo, Áreas verdes del municipio de Monterrey, Universidad Autónoma de Nuevo Léon, Facultad de Ciencias Florestales, Ayuntamiento de Monterrey, Oct. 2013, p.1-21.

PÉREZ-MEDINA Susana, LÓPEZ-FALFÁN Ina, « Áreas verdes y arbolado en Mérida, Yucatán. Hacia una sostenibilidad urbana », Sociedad y Territorio, vol.XV, n47, 2015, p.1-33.

PRATES Marco, Curitiba é capital com mais carros por pessoa-veja ranking, 2014, https:// exame.abril.com.br/brasil/curitiba-e-capital-com-mais-carros-por-pessoa-veja-ranking/.

RAPPORT BRUNDTLAND, Avant-propos, Notre avenir à tous, Avant-propos de la présidente, 2018, https://www.diplomatie.gouv.fr/sites/odyssee-developpement-durable/files/5/ rapport_brundtland.pdf, 2018.

RIVAS TORRES Daniel, Planeación, espacios verdes y sustentabilidad en el Distrito Federal. Universidad Autónoma Metropolitana, Tesis de doctor en diseño, 2005, http://www.rivasdaniel.com/ Espaciosverdes.pdf.

SABOIA Luciana, « Brasilia et Oscar Niemeyer : le contexte politique et la dimension esthétique », Cahiers d'Histoire, n¹09, 2009, p.27-54.

SÁNCHEZ RUIZ Gerardo, Planificación y Urbanismo de la Revolución Mexicana, Universidad Autónoma Metropolitana Azcapotzalco-Asamblea Legislativa del Distrito Federal, México, Distrito Federal, 2002.

SÁNCHEZ RUIZ Geraldo, «El arquitecto Carlos Contreras y la construcción de las bases para el desenvolvimiento de la ciudad de México. Los años 1925 a 1934 », Diseño en Síntesis, UAMAzcapotzalco, México, n¹3, 2002.

SÁNCHEZ RUIZ Gerardo (dir.), « Planificación y Urbanismo visionarios de Carlos Contreras, escritos de 1925 a 1938 », Colección Raíces, n² 2, 2003.

SOARES Antonio Carlos Lobo, Impactos da urbanizacao sobre parques públicos : estudo de caso do parque zoobotanico do Museu Goeldi (Belém -PA), Dissertacao, Programa de Mestrado em Desenvolvimento e Meio Ambiente Urbano da Universidade da Amazonia, Belém. 2009, http:// repositorio.museu-goeldi.br/bitstream/mgoeldi/1233/1. 
TONDE Alfred Jonas, Rôle, importance et gestion des espaces verts dans la ville de Ouagadougou, Mémoire de maîtrise, 1994, http://www.beep.ird.fr.

LONG Nathalie, TONINI Brice, « Les espaces verts urbains : étude exploratoire des pratiques et du ressenti des usagers ", Vertigo, vol.12, $\mathrm{n}^{\circ} 2$, sept. 2012, https://journals.openedition.org/vertigo/ 12931

VIZCAYA Isidro, Los orígenes de la industrialización de Monterrey. Una historia económica y social desde la caída del segundo imperio hasta el fin de La revolución 1867-1920, Monterrey, Fondo Editorial Nuevo León e Instituto Tecnológico de Monterrey, 2006.

ZAMORA Elia Canosa, POMBO, SAEZ Ester, BRASSART, SANABRIA Cristina, MORENCO Zavala Ignácio, "Metodología para el estudio de los parques urbanos : la Comunidad de Madrid », Geofocus: Revista Internacional de Ciencia y Tecnología de la Información Geográfica, n³, 2003.

\section{NOTES}

1. Índice de cidades verdes da américa latina, 2010.

2. AURICOSTE, Isabelle, Urbanisme moderne et symbolique du gazon, 2003, p.25.

3. PAQUOT Thierry, Ville et nature, un rendez-vous manqué ?, 2004, p.84.

4. AURICOSTE Isabelle, Urbanisme moderne et symbolique du gazon, 2003, p.19.

5. MONTIGNY Gilles, De la ville à l'urbanisation, Éditions L'Harmattan, 1992.

6. PAQUOT Thierry, Ville..., op.cit., 2004, p.89.

7. DUBOST Françoise, LIZET Bernadette, «La nature dans la cité », Communications, 74, 2003, p. 6.

8. MONCEF Btissam, CARBONE Valentine, SOULEROT Marion, Le management durable au cour des organisations, Paris, Lavoisier, 2011, p.214.

9. MONGIN Olivier, Conférence sur l'élaboration du plan local d'urbanisme. Intégral du discours de M. Mongin, tenu le 6 octobre 2010 à l'Hôtel de Ville de Neuilly-sur-Seine, 2010.

10. RAPPORT BRUNDTLAND, « Avant-propos, Notre avenir à tous », 2018, p.342.

11. MONGIN Olivier, Conférence..., op. cit., p.1.

12. BANZO Mayté, Structure verte et mise en cohérence de la périphérie métropolitaine de Lisbonne, 2007.

13. BANZO Mayté, Ibidem, p.114.

14. ANTONI Robert Max (dir.), «De l'espace public», Séminaire Robert Auzelle, Art Public, Ministère du Logement et de l'habitat durable 2015, p.105.

15. TONDE Alfred Jonas, Rôle et importance et gestion des espaces verts dans la ville de Ouagadougou, Mémoire de maitrise, 1994, p.29.

16. MEILLERAND Marie-Clotilde, Les espaces verts dans les plans d'urbanisme de l'agglomération lyonnaise : des espaces verts à la ceinture verte, Lyon, ENS-LSH, Séminaire GRAIQUE, 4 mars. 2008.

17. MEILLERAND Marie-Clotilde, Ibidem, p.8.

18. Le premier plan date de 1875, BERDOULAY, Vincent. Modernismo e espaço público: o Plano Agache do Rio de Janeiro, 2003.

19. Le pays a remplacé le régime politique républicain par la dictature du président Getúlio Vargas.

20. "Ainda que poucas realizações concretas tenham decorrido desse plano, sua influência perdurou pelas ideias que ele permitiu discutir (como a de zoneamento) e pelo primeiro grande código de obras editado em 1937 a partir do mesmo, que continua a inspirar a legislação urbanística atual », BERDOULAY Vincent, Ibidem, p.124.

21. "Aún así, muchas de sus proposiciones, aunque fragmentadas, fueron aprovechadas en la nueva legislación urbanística aprobada en 1937 (Decreto 6000) y en los estudios posteriores desarrollados por el 
Servicio Técnico del Plano de la Ciudad dirigido por José de Oliveira Reis. Es el caso, por ejemplo, de la urbanización del Jardín de Alá, nítidamente inspirada en las propuestas agachianas », FARIA Renata Pereira. Plan Agache de Rio de Janeiro, capital federal du Bresil: urbanismo de excelencia en los años 20, 2006, p.81.

22. LUCIO COSTA, « Le véritable père de Brasilia », Lumière de la ville, 2016.

23. SABOIA Luciana, Brasilia et Oscar Niemeyer: le contexte politique et la dimension esthétique, 2009, p. 42.

24. DUBARLE Patrick, PELLEGRIN Jean-Pierre, Politiques et pratiques d'aménagement du territoire au Brésil.

25. LONG Nathalie, TONINI Brice, Les espaces verts urbains : étude exploratoire des pratiques et du ressenti des usagers, 2012, p.1.

26. "El concepto de 'ciudades verdes' -con capacidad de recuperación, autosuficiencia y sostenibilidad social, económica y ambiental- se suele asociar a la planificación urbana en los países más desarrollados. Indica una eco-arquitectura de alta tecnología, rutas arboladas para las bicicletas e industrias de 'circuito cerrado' que no producen desechos. Sin embargo, tiene una aplicación especial y dimensiones sociales y económicas considerablemente diferentes en los países en desarrollo de bajos ingresos. Allí, los principios centrales de unas ciudades más verdes pueden orientar un desarrollo urbano que garantice la seguridad alimentaria, un trabajo e ingresos dignos y una buena gobernanza para todos los ciudadanos" (Horticultura urbana y periurbana. organización de las naciones unidas para la alimentación y la agricultura. ciudades más verdes ¿ Ciudades de sufrimiento o de oportunidades ?, 2018).

27. Emplasa. Empresa paulista de planejamento metropolitano. Sobre a RMSP. Disponible à l'adresse: https://www.emplasa.sp.gov.br/RMSP.

28. «Considera-se área verde de domínio público, para efeito desta Resolução, o espaço de domínio público que desempenhe função ecológica, paisagística e recreativa, propiciando a melhoria da qualidade estética, funcional e ambiental da cidade, sendo dotado de vegetação e espaços livres de impermeabilização. "

29. NASCIMENTO Karina Barros Freitas, Áreas verdes e clima urbano: a função socioambiental dos imóveis de proteção de áreas verdes na cidade - Recife/PE, Brasil, Dissertação (Mestrado), Programa de Pós-Graduação em Desenvolvimento Urbano, Universidade Federal de Pernambuco, Recife, 2011.

30. PINTO Monica, Ambiente Brasil Exclusivo : Curitiba tem a primeira Reserva Particular do Patrimônio Natural Municipal - RPPNM do país, 2007. A RPPNM Cascatinha nasceu do esforço de moradores do bairro de Santa Felicidade, na zona oeste de Curitiba. "Aqui nós temos uma característica ímpar, de união entre os canais de comunicação e representação em prol dos interesses do bairro", diz o proprietário da reserva, Eurico Borges dos Reis, morador há cerca de 30 anos ».

31. DOURADO Anisio Brasileiro de Freitas, «Développement urbain et transports collectifs au Brésil. Les enseignements de l'expérience de Recife et de Curitiba », Sociologie, 1991, p.283.

32. « A decisão da prefeitura de Curitiba de reajustar a tarifa do transporte coletivo com índice maior do que o dobro da inflação deve dificultar ainda mais a tarefa de reverter a tendência de queda no total de passageiros transportados pelo sistema da cidade. De 2011 para 2016, o total de passageiros pagantes caiu de 246,8 milhões por ano para 211 milhões - redução de 20\%. E a projeção é de nova queda para 2017 ». MARCHIORI Raphael, BORDIN Laura Beal, «Em 5 anos, Curitiba perdeu 20\% dos passageiros de ônibus; tendência é piorar ", Gazeta do Povo, 2017.

33. Uber est une application mobile qui met en relation directe les utilisateurs avec des voitures de tourisme avec chauffeur. Voir https://fr.softonic.com/articles/uber-vtc-cest-quoi.

34. PRATES Marco, Curitiba é capital com mais carros por pessoa-veja ranking. 2014.

35. BAZANI Adamo, "Volvo confirma a entrega de 25 novos biarticulados para Curitiba em março », Diário do Transporte, 2017.

36. "Obra do Ligeirão começa nesta segunda e deve deixar trânsito lento na Praça do Japão ", Gazeta do Povo, 2018. 
37. «Com faixas com o slogan 'S.O.S Praça do Japão' e balóes vermelhos nas mãos, moradores do entorno da Praça do Japão, em Curitiba, se juntaram em um abraço simbólico no local nesta manhã de domingo (18). A manifestação tem o objetivo de chamar a atenção para as alterações causadas pela implantação da nova linha Ligeirão no local. De acordo com os manifestantes, a mudança irá complicar o acesso à praça e tornála mais perigosa principalmente para os idosos e crianças que costumam frequentá-la ».

38. SOARES Antonio Carlos Lobo, Impactos da urbanizacao sobre parques públicos: estudo de caso do parque zoobotanico do Museu Goeldi (Belém -PA), Dissertacao, Programa de Mestrado em Desenvolvimento e Meio Ambiente Urbano da Universidade da Amazonia, Belém, 2009.

39. « Em 1977, a vegetação do parque já estava isolada de outros maciços verdes encontrados no centro das quadras, correspondendo aos quintais dos lotes com frente para as vias. Assinalam-se, em 1998, os quatro edifícios na Tv. Nove de Janeiro e um na Av. Alcindo Cacela, que passaram a sombrear o PZB (Parque Zoobotanico)». Ibidem, p.109.

40. «Essa participação inferior a 40\% em um primeiro momento reforça a informação dos jornais da época de que a maior parte da população estava indiferente ao processo decisório de mudança, demonstrando mais uma vez a apatia e complacência da maioria da população curitibana diante das mudanças realizadas pela prefeitura ». HASSLER Márcio Luís, A natureza na cidade: Uma abordagem a partir da percepção da população acerca do Jardim Botânico de Curitiba-PR, Dissertacao, Curso de Mestrado em Geografia, Universidade Federal do Paraná, 2006, p.77.

41. "Curitiba - Pouco depois que a Prefeitura de Curitiba iniciou as obras para dividir ao meio a Praça Miguel Couto, a Pracinha do Batel, ontem de manhã, cerca de 50 moradores e comerciantes se dirigiram ao local para impedir o trabalho das motosserras e tratores. Entre eles estava Rafael Xavier, de 26 anos, bacharel em Direito e morador, há dez anos, das redondezas. o jovem se colocou diante do trator e as outras pessoas se posicionaram na frente das motosserras para evitar que a praça centenária fosse demolida». MORADORES IMPEDEM OBRAS NA PRAÇA DO BATEL, Folha de Londrina, 2007.

42. Ibidem.

43. FIGUEROA Jonás, « La arquitectura y el urbanismo en la América Latina del siglo XX », dans EN PUNCEL Alfonso (Ed.), Las ciudades de América Latina: problemas y oportunidades. Universitat de Valencia, 1994.

44. GUTIÉRREZ C.J.J, «Planeación urbana en México: un análisis crítico sobre su proceso de evolución urbano », Universidad del Bío Bío, Vol.12, n¹9, 2009, p.55.

45. DAVIS Diane E, El Leviatán urbano. La ciudad de México en el siglo XX. México, Distrito Federal: Fondo de Cultura Económica, 1999.

46. Inégalités et urbanisation. 2016. Disponible à l'adresse : http://www.oecd.org/fr/regional/ inegalites-et-urbanisation.htm.

47. KRISHNAMURTHY L., NASCIMENTO José Rente (eds.). Áreas Verdes Urbanas en Latinoamérica y el Caribe, 1998. Disponible à l'adresse : www.rivasdaniel.com/Pdfs/Areas_verdes_LatAmerica.pdf.

48. BURDETT Ricky, Inégalités et urbanisation, 2016, p. 1.

49. Estudios de Políticas Urbanas de la OCDE: México Transformando la Política Urbana y el Financiamiento de la Vivienda, 2015.

50. Instituto Nacional de Estadística y Geografía, Principales resultados del Censo de Población y Vivienda 2010.

51. GUTIERREZ C.J.J., « Planeación ... », op.cit., 2009.

52. Le bassin endoréique verse ses eaux superficielles ou non dans une dépression fermée. Alors, l'eau y retenue peut sortir seulement par infiltration ou évaporation.

53. RIVAS TORRES Daniel, Planeación, espacios verdes y sustentabilidad en el Distrito Federal. Universidad Autónoma Metropolitana. Tesis de doctor en diseño. 2005, p.2.

54. MCMICHAEL C., "The Urban Development of Mexico City, 1850, 1930 », dans ALMANDOZ Arturo (Ed.). Planning Latin America's Cities, 1850-1950, Londres, Routledge, 2002.

55. DAVIS Diane E, El Leviatán urbano. La ciudad de México en el siglo XX. México, Distrito Federal: Fondo de Cultura Económica, 1999. 
56. SÁNCHEZ RUIZ Gerardo, Planificación y Urbanismo de la Revolución Mexicana, Universidad Autónoma Metropolitana Azcapotzalco-Asamblea Legislativa del Distrito Federal, México, Distrito Federal, 2002; El arquitecto Carlos Contreras y la construcción de las bases para el desenvolvimiento de la ciudad de México. Los años 1925 a 1934, Diseño en Síntesis, UAM-Azcapotzalco, México, 2002, n¹3 ; «Planificación y Urbanismo visionarios de Carlos Contreras, escritos de 1925 a 1938 ", Colección Raíces, n². Universidad Nacional Autónoma de México, Universidad Autónoma Metropolitana Azcapotzalco y Universidad Autónoma de San Luis Potosí, 2003.

57. GUTIERREZ C.J.J., « Planeación ... », op.cit., 2009.

58. SÁNCHEZ RUIZ, cité dans RIVAS TORRES Daniel, Planeación, espacios verdes y sustentabilidad en el Distrito Federal, Universidad Autónoma Metropolitana. Tesis de doctor en diseño, 2005, p.2.

59. «Las propuestas de traza de Contreras para el Distrito Federal fueron, con los años, adoptándose: los circuitos interior y exterior, las grandes avenidas que lo cruzaban (por ejemplo, los ejes viales), la main street, que iba a ser la avenida San Juan de Letrán (de Tlalnepantla al arranque de la carretera a Cuernavaca) que terminó siendo Insurgentes, el metro del cual hizo, en 1949, los primeras negociaciones y trazos, y la urbanización El Pedregal, obra del arquitecto Luis Barragán, del cual dibujó el fraccionamiento sobre fotografías aéreas », ESCUDERO Alejandrina, «Carlos Contreras... », op.cit.,2007, p.4.

60. GUTIÉRREZ C.J.J., « Planeación ... », op.cit., 2009.

61. Offre des logements compatibles au pouvoir d'achat de chaque personne.

62. GUTIÉRREZ C.J.J., « Planeación ... », op.cit., 2009.

63. GUTIÉRREZ, C.J.J., Ibidem.

64. «En 1988, se produjo un nuevo desarrollo legislativo en la tendencia de acumulación normativa que venimos señalando. Se trata de la Ley General del Equilibrio Ecológico y la Protección al Ambiente (LGEEPA), que constituye el primer intento de regular la cuestión ambiental en su conjunto, desde los efectos de las actividades industriales hasta la operación de las áreas naturales protegidas. Una vez más, con la pretensión de la integralidad ». AZUELA Antonio, «Sobre las nuevas condiciones en el acceso al suelo y en la regulación urbana en México ", dans FERNANDES Edésio, ALFONSIN Betânia (coord.), Direito urbanístico. Estudos brasileiros e internacionais, Belo Horizonte: Del Rey, 2006, p.132.

65. ZAMORA Elia Canosa, POMBO Ester Sáez, BRASSART S., SANABRIA Sanabria, MORENCO Zavala Ignácio, "Metodología para el estudio de los parques urbanos: la Comunidad de Madrid", Geofocus : Revista Internacional de Ciencia y Tecnología de la Información Geográfica, n³, 2003.

66. PÉREZ-MEDINA Susana, LÓPEZ-FALFÁN Ina, « Áreas verdes y arbolado en Mérida, Yucatán. Hacia una sostenibilidad urbana ", Sociedad y Territorio, Vol.XV, n47, p.1-33, 2015.

67. "Por lo tanto, en la ciudad de Monterrey se cuenta con $6.2 \mathrm{~m} 2$ de área verde por habitante. Este resultado no puede ser interpretado como superior a los $4.8 \mathrm{~m} 2$ reportados por Roccatti (2004) ya que debe tenerse en cuenta que la mayoría de las áreas verdes se concentran en el sur y poniente de Monterrey, lo que las torna en zonas prácticamente inaccesibles para los habitantes del centro y norte de la ciudad", PÉREZ Javier Jiménez, CUÉLLAR Geraldo, TREVIÑO Eduardo, Áreas verdes del municipio de Monterrey, Universidad Autónoma de Nuevo Léon, Facultad de Ciencias Florestales, Ayuntamiento de Monterrey, Oct. 2013, p.1-21.

68. «Sin embargo, su configuración inicial y la falta de un plan de desarrollo inicial hacen prácticamente imposible la creación de nuevas áreas verdes ", Ibidem, p.7.

69. SÁNCHEZ RUIZ Gerardo, Ibidem.

70. «Para lograr una de las finalidades de la planificación se consideraba necesaria alguna forma de control sobre la propiedad pública y privada. Hasta ese momento sólo se contaba con una primera ley para Monterrey, preparada por Carlos Contreras, cuando Aarón Sáenz era gobernador de Nuevo León.» ESCUDERO Alejandrina, SÁNCHEZ RUIZ, Gerardo G GARZA, Carlos Ríos, "Estudios Introductorios », Revista Planificación (1927-1936), Universidad Nacional Autónoma de México, 2018, p.49. 
71. «Los ríos servían a la vez como vertedero de desechos; muchos ríos desaparecieron por la actividad industrial, como el río Santa Catarina en Monterrey ». La ville de Monterrey, en 1930 avait une population de 148.000 personnes et un territoire de 1.780 hectares, PEREZ DANIEL Gustavo Héron, Estudios de Historia Moderna y Contemporánea de México, nº 42, julio-diciembre 2011, p.83.

72. ESPARZA HERNÁNDEZ Luis Gerardo, Estudio del manejo y conservación del Parque Nacional Cumbres de Monterrey (PNCM), según decreto del 2000 bajo un enfoque de desarrollo sustentable basado en la participación ciudadana. Tesis. Universidad Autónoma de Nuevo León. Instituto de Investigaciones Sociales, 2014.

73. CASILLAS ZAPATA Amanda Melissa, ELIZONDO María Teresa Ledesma, MORENO Carlos Estuardo Aparicio, "Conformación de áreas verdes y espacios abiertos en la transformación urbana de Monterrey del siglo XVII a inicios del siglo XXI », Letras Históricas, 2018, p.1-32.

74. «[Él] inauguró el paseo Santa Lucía en el mes de septiembre de 2007; sobresale la construcción del pabellón ciudadano y un canal artificial de agua clorada con una capacidad de 12'000,000 de litros simulando el río Santa Lucía, que en parte quedó sepultado por el canal y por la plancha de concreto de la macroplaza construida durante el mandato del gobernador el C. Alfonso Martínez Domínguez ", ESPARZA HERNANDEZ Luis Gerardo, Estudio..., op.cit., 2014, p.149.

75. « El M.C. Rodrigo Medina de la Cruz no ha creado hasta el momento nuevas áreas naturales protegidas como sus antecesores, pero el 21 de diciembre de 2011 publicó en el Diario Oficial el decreto de ampliación del parque urbano La Pastora que aumentará en 13.65 ha ubicadas fundamentalmente en el lecho del río La Silla, el único río vivo en el AMM. Este parque será administrado y manejado por un consejo ciudadano. Al poco tiempo le otorgó en concesión a FEMSA-Coca Cola una superficie de 26 ha de esta zona por 60 años para construir el estadio de futbol del Club Los Rayados, concesión que no fue posible revertir por el movimiento ecologista (Figura 68) pese a que argumenta graves daños al río La Silla y el aumento del déficit de áreas verdes ", Ibidem, p.151.

76. EL HORIZONTE, Acaparan privados áreas verdes de Parque Fundidora. Los directivos del parque estatal elevaron la frecuencia de eventos privados en la zona de las $S$ 's, que es su principal área verde, 2016; Parque Fundidora hace elitista diversión familiar. Con precios elevados, proveedoras de entretenimiento hacen su agosto a costa de la economía familiar, 2014.

77. Punto de acuerdo para solicitar comparecencia de director del parque fundidora. Disponible à l'adresse: http://www.hcnl.gob.mx/glpvem/2016/10/punto-de-acuerdo-para-solicitarcomparecencia-de-director-del-parque-fundidora.php.

78. Expression datée du XVII ${ }^{e}$ siècle, qui fait référence aux combats de lutte sur le sable ou la terre. Le perdant était celui qui était à terre.

\section{RÉSUMÉS}

Quoique l'image du «vert naturel» demeure un élément important du paysage urbain, la fonction des espaces verts a changé de même que les termes qui les désigne. En prenant appui sur deux études de cas, Curitiba au Brésil et Monterrey au Mexique, cet article analyse les conflits en cours autour de la conception des espaces verts urbains. Les choix politiques pour les redynamiser et les équiper en infrastructures et constructions conduisent (1) à leur transformation en espace de consommation, ce qui peut avoir des conséquences néfastes pour la ville, ou (2) à une réduction de leur rôle récréatif et écologique, pour la gestion de l'eau, de l'air, de la température et de la biodiversité. En croisant références bibliographiques et informations 
recueillies lors des visites in situ, nous sommes arrivés à cette conclusion que les espaces verts urbains sont actuellement menacés de disparition et cela alors qu'ils devraient représenter un patrimoine environnemental et faire partie des objectifs de la soutenabilité. La bataille continue pour renverser la tendance à ne plus les concevoir comme espace public, à les réduire en taille ou à les éliminer du tissu urbain.

The twentieth century town planning is strongly linked to territory development. For city technician designers, the environmental flap was first present in the form of squares, parks and gardens. Thus, the modern came, carrying the hygienist ideology, a restorative virtue of health and well-being, and at the same time, the mutation of human nature. Despite the image of natural green remained as an important element of the urban landscape, the functions of these spaces have changed and also the terms that named them, some recovered from biology, such as ecology, sustainable green spaces and other. The context of this article takes up the political conflicts regarding urban green spaces' design concepts as public spaces appropriate for citizens. The political choices to revitalize and equip them with infrastructures and constructions, punctuated by agreements with the private sector, present two views on the urban windows : (1) transformation of the green space into consumption space, which can have harmful consequences for the city, or (2) burn to ashes of their amusement role, water supply, air, temperature, and biodiversity. Who are the political actors responsible for maintaining its value, cleanliness and usefulness? To reflect on this question, as by looking through historical windows and visual sequences, we rely on two case studies: Curitiba in Brazil, and Monterrey in Mexico. Using the bibliographic references obtained, and compilation of information collected by the authors, during visits to the sites and learning to look at the city, we conclude that the roles of public green spaces available in cities are more defined today by its different functions, and that they are threatened with extinction and no longer being part of ecosystems. We believe that although green spaces are more associated with public and private interests, maintenance and amusement than with hygienist thinking and the macrocosm of human nature, they may be part of the goals of sustainability and represent an environmental heritage. The battle continues to reverse the political trends of no longer conceiving them as public space, to reduce them in size or to eliminate them from the urban space.

\section{INDEX}

Mots-clés : histoire des techniques, urbanisme, ville, soutenabilité, patrimoine environnemental Keywords : history of technology, urban planning, town, sustainability, urban ecosystem, environmental heritage

Thèmes : Horizons internationaux

\section{AUTEURS}

\section{MACLOVIA CORRÊA-DA-SILVA}

Titulaire d'un doctorat en Structures Environnementales et Urbaines de la Faculté d'Architecture et Urbanisme de l'Université de Sao Paulo et d'une maîtrise en Histoire du Brésil de l'Université Fédérale de Paraná, Maclovia Corrêa da Silva est depuis 1994 professeur à l'Université Technologique Fédérale du Paraná, à Curitiba (Brésil), dans le cadre du Programme de Master et Doctorat en Technologie et Société (UTFPR-PPGTE-Brésil). Elle est référente pour l'UTFPr du master Erasmus Mundus TPTI, dont elle a été Visiting Scholar à l'Université Paris 1 Panthéon Sorbonne. Elle participe au programme de recherche Technologie et Développement de l'UTFPr. 


\section{MARIA EUGENIA GONZÁLEZ-ÁVILA}

Docteur en Gestion et Utilisation des Ressources Naturelles par le Centre de Recherche Biologique du Nord-est (CIBNOR), Maria Eugenia Gonzalez-Ávila est biologiste à l'Université Nationale Autonome du Mexique (UNAM) à Mexico. Depuis 2007, elle est professeur-chercheur titulaire "B" et actuellement coordinatrice du programme de maîtrise en gestion intégrée de l'eau au Collège de la Frontière Nord (Monterrey). Ses domaines de recherche sont les impacts environnementaux transfrontaliers et la durabilité urbaine avec un accent mis sur l'eau, l'énergie et les déchets. En 2009, elle a été distinguée par le Fellow El Colegio de México du programme LEAD-Mexico Generation 14. Elle appartient actuellement au Système national de chercheurs, niveau 1, et est un évaluateur agréé : RCEA-05-00002-2008. 